\title{
Event and Map Content Personalisation in a Mobile and Context-Aware-Environment
}

\author{
Ralf Bierig \\ School of Computing \\ The Robert Gordon University \\ Aberdeen, Scotland, UK \\ ralf@bierig.net \\ http://www.bierig.net
}

\begin{abstract}
Effective methods for information access are of the greatest importance for our modern lives - particularly with respect to handheld devices. Personalisation is one such method which models a user's characteristics to deliver content more focused to the user's needs. The emerging area of sophisticated mobile computing devices has started to inspire new forms of personalised systems that include aspects of the person's contextual environment. This thesis seeks to understand the role of personalisation and context, to evaluate the effectiveness of context for content personalisation and to investigate the event and map content domain for mobile usage. The work presented in this thesis has three parts:

The first part is a user experiment on context that investigated the contextual attributes of time, location and interest, with respect to participants' perception of their usefulness. Results show highly dynamic and interconnected effects of context on participants' usefulness ratings.

In the second part, these results were applied to create a predictive model of context that was related to attribution theory and then combined with an information retrieval score to create a weighted personalisation model.

In the third part of this work, the personalisation model was applied in a mobile experiment. Participants solved situational search tasks using a (i) non-personalized and a (ii) personalized mobile information system, and rating entertainment events based on usefulness. Results showed that the personalised system delivered about $20 \%$ more useful content to the mobile user than the non-personalised system, with some indication for reduced search effort in terms of time and the amount of queries per task.

The work presented provides evidence for the promising potential of context to facilitate personalised information delivery to users of mobile devices. Overall, it serves as an example of an investigation into the effectiveness of context from multiple angles and provides a potential link to some of the aspects of psychology as a potential source for a deeper understanding of contextual processes in humans.
\end{abstract}

